\title{
Ensayo randomizado del uso de pesario cervical para prevenir parto prematuro en embarazos únicos (1)
}

\author{
Nicolaides KH, Syngelaki A, Poon LC, Picciarelli G, Tul N, Zamprakou A, Skyfta E, Parra- \\ Cordero M, Palma-Dias R, Rodriguez-Calvo J. A randomized trial of a cervical pessary \\ to prevent preterm singleton birth. N Engl J Med. 2016: 374(11):1044-52.
}

Análisis Crítico: Rafael Valdés V., Jorge A Carvajal C. PhD.

Unidad de Medicina Materno-Fetal, División de Obstetricia y Ginecología, Escuela de Medicina, Pontificia Universidad Católica de Chile.

\section{RESUMEN (1)}

Introducción: La prematurez es la principal causa de mortalidad y morbilidad neonatal e infantil. No está claro si un pesario insertado alrededor del cuello del útero, reduce el riesgo de parto prematuro en embarazos únicos. Método: Condujimos un estudio multicéntrico, aleatorizado y controlado que compara la colocación del pesario, con el manejo expectante (control) en mujeres con embarazos únicos y que tenían una longitud del cuello del útero de $25 \mathrm{~mm}$ o menos, entre las 20 semanas y 0 días y 24 semanas y 6 días de gestación. Los participantes de ambos grupos que tenían una longitud cervical de $15 \mathrm{~mm}$ o menos en la aleatorización o en visitas posteriores, recibieron tratamiento con progesterona vaginal. El resultado primario fue parto espontáneo antes de las 34 semanas de embarazo. Resultados: En un análisis por intención de tratar, no hubo diferencia significativa entre el grupo pesario (465 participantes) y el grupo control (467 participantes) en la tasa de parto espontáneo antes de las 34 semanas $(12,0 \%$ y $10,8 \%$, respectivamente); OR 1,12 (en el grupo pesario) IC95\% 0,75 a 1,69; $p=0,57$. No hubo diferencias significativas en las tasas de mortalidad perinatal $(3,2 \%$ en el grupo pesario y el $2,4 \%$ en el grupo control, $p=0,42)$, el resultado neonatal adverso $(6,7 \%$ y $5,7 \%$, respectivamente; $p=0,55)$, o neonatal con cuidados especiales $(11,6 \%$ y $12,9 \%$, respectivamente; $p=0,59)$. La incidencia de aumento de flujo vaginal fue significativamente mayor en el grupo pesario que en el grupo control. Conclusiones: En mujeres con embarazos únicos que tenían un cuello uterino corto, un pesario cervical no se tradujo en una menor tasa de parto prematuro espontáneo temprano, comparado con conducta expectante.

\section{ANÁLISIS DE LA INVESTIGACIÓN}

\section{A. Relevancia clínica de la investigación}

El parto prematuro es responsable de más del $70 \%$ de todas las muertes neonatales e infantiles. Además, el riesgo de parálisis cerebral en niños prematuros es 10 veces mayor que entre los nacidos a término (2). Los riesgos de mortalidad y morbilidad perinatal están inversamente relacionados con la edad gestacional al nacimiento. El riesgo de parto prematuro está inversamente relacionado con la longitud cervical, medido por ecografía entre las 20 y 24 semanas. Ensayos randomizados, controlados, aleatorios han demostrado que el uso profiláctico de progesterona disminuye significativamente la tasa de parto prematuro y muerte neonatal en mujeres con embarazos únicos y que tenían una longitud cervical menor a $15 \mathrm{~mm}$, en comparación a aquellas que no recibieron progesterona (3-4). Metaanálisis de cerclaje cervical realizados en mujeres con embarazos únicos y con cuello uterino corto, no han mostrado una tasa significativamente menor de parto prematuro en general que la tasa sin cerclaje, aunque han mostrado beneficio en el subgrupo de mujeres que tenían un parto prematuro previo (5$6)$. Un enfoque alternativo para la prevención del 
parto prematuro es la colocación de un pesario de silicona alrededor del cuello del útero; este dispositivo está pensado para soportar la presión sobre el cuello uterino y cambiar su dirección hacia el sacro, reduciendo así la presión directa del embarazo sobre el canal cervical. Dos ensayos aleatorios en mujeres con embarazos únicos que tenían cuello uterino corto han sido publicados (después del inicio de este estudio), proporcionado resultados contradictorios sobre el efecto en la tasa de parto espontáneo antes de las 34 semanas; es un ensayo que incluyó 380 mujeres, la tasa de este resultado fue significativamente menor con pesario que sin pesario (6\% vs. $27 \%$ ), pero en el segundo ensayo, que incluyó 108 mujeres, no hubo efecto significativo $(9,4 \%$ y $5,5 \%$, respectivamente) $(7,8)$.

\section{B. El estudio (1)}

Diseño: Estudio multicéntrico, randomizado y controlado que compara la colocación del pesario con el manejo expectante (control) en mujeres con embarazos únicos, en 16 maternidades de Inglaterra, Alemania, Eslovenia, Portugal, Australia, Italia, Albania, Bélgica y Chile. Pacientes: mujeres > 16 años de edad, con embarazos único, que se sometieron a examen ecográfico de rutina entre las $20+0$ semanas y 24+6 semanas de gestación, y que tenían una cervicometría $<25 \mathrm{~mm} ; 465$ pacientes en grupo pesario y 467 grupo control. Intervención: Instalación del pesario vaginal, al momento de la detección del cuello corto. Comparación: Vigilancia, sin uso del pesario. En pacientes con cervicometría $<15 \mathrm{~mm}$, se utilizó progesterona natural $(200 \mathrm{mg}$ ) hasta las 33+6 semanas de embarazo. Resultado primario: Parto espontáneo antes de las 34 semanas (238 días) de gestación. Resultado secundario: Peso al nacer (media, $<2,5 \mathrm{~kg}$ y $<1,5 \mathrm{~kg}$ ), muerte perinatal (fetal o neonatal), una combinación de eventos adversos en el recién nacido antes del alta hospitalaria (cualquier grado de hemorragia intraventricular, síndrome de dificultad respiratoria, retinopatía del prematuro o enterocolitis necrotizante), indicador compuesto de cuidados especiales neonatales (ingreso en la unidad de cuidados intensivos neonatales, ventilación mecánica, fototerapia, tratamiento de la sepsis sospecha o confirmada, transfusión sanguínea), y complicaciones maternas atribuibles a pesario (muerte materna, grave traumatismo cervical o vaginal, corioamnionitis).

\section{Análisis Crítico}

Validez interna: Estudio con descripción apropiada de los criterios de inclusión y exclusión, multicéntrico, randomizado, ciego, con ocultamiento de la secuencia de randomización, similitud de pacientes en ambos grupos, con seguimiento completo y análisis por intención de tratar. Existen varias limitaciones: en primer lugar, sólo 935 participantes (58\%) del previsto inicialmente, 1600 se sometieron a la aleatorización, lo que aumenta el riesgo de error al azar. Sin embargo, el número reclutado es considerablemente superior a los 380 y 108 participantes en los dos ensayos aleatorizados previos de la colocación del pesario en mujeres con embarazos únicos con riesgo de parto prematuro. En segundo lugar, la distribución observada de longitud cervical difiere de la utilizada para los cálculos de potencia (29\% y $71 \%$ para las respectivas longitudes de 1 a $15 \mathrm{~mm}$ y de 16 a $25 \mathrm{~mm}$, en lugar de $14 \%$ y $86 \%$ ). Se retiró el pesario a un $10 \%$ de la población estudiada por molestias asociadas a su uso; esto sin embargo, no afecta el resultado del estudio, pues el análisis fue por intención de tratar.

Validez externa: El estudio es aplicable a nuestra población, y los pesarios están disponibles en nuestro medio; del mismo modo, la progesterona está ampliamente disponible en nuestro país en aquellas pacientes que lo requieran. Adicionalmente, se incluyó pacientes chilenas en el estudio.

Conclusiones: Estudio con metodología adecuada, pero con elevado riesgo de sesgo por no completar el tamaño muestral calculado, lo que aumenta las probabilidades de error al azar y reduce el poder del estudio. La información de este estudio, asociado a los resultados de otras investigaciones similares, sugiere que el pesario vaginal no debe ser utilizado como herramienta de prevención del parto prematuro en mujeres con cuello corto.

\section{REFERENCIAS}

1. Nicolaides $\mathrm{KH}$, Syngelaki A, Poon LC, Picciarelli G, Tul N, Zamprakou A, Skyfta E, Parra-Cordero M, Palma-Dias $\mathrm{R}$, Rodriguez-Calvo J. A randomized trial of a cervical pessary to prevent preterm singleton birth. N Engl J Med. 2016;374(11):1044-52.

2. Saigal S, Doyle LW. An overview of mortality and sequelae of preterm birth from infancy to adulthood. Lancet 2008;371:261-9.

3. Fonseca EB, Celik E, Parra M, Singh M, Nicolaides $\mathrm{KH}$. Progesterone and the risk of preterm birth among women with a short cervix. N Engl J Med 2007;357:462-9.

4. Romero R, Nicolaides K, Conde- Agudelo A, et al. Vaginal progesterone in women with an asymptomatic sonographic short cervix in the midtrimester decreases preterm delivery and neonatal morbidity: a systematic review and metaanalysis of individual patient data. Am J Obstet Gynecol 2012;206(2):124.e1-19.

5. Berghella V, Odibo AO, To MS, Rust OA, Althuisius SM. Cerclage for short cervix on ultrasonography: meta-analysis of trials using individual patient-level data. Obstet Gynecol 2005;106:181-9.

6. Berghella V, Rafael TJ, Szychowski JM, Rust OA, Owen J. Cerclage for short cervix on ultrasonography in women with singleton gestations and previous 
preterm birth: a meta-analysis. Obstet Gyneco 2011;117:663-71.

7. Arabin B, Halbesma JR, Vork F, Hübener M, van Eyck $\mathrm{J}$. Is treatment with vaginal pessaries an option in patients with a sonographically detected short cervix? J Perinat Med 2003;31:122-33.
8. Goya M, Pratcorona L, Merced C, et al. Cervical pessary in pregnant women with a short cervix (PECEP): an open-label randomised controlled trial. Lancet 2012; 379:1800-6. 